Article

\title{
Tra saeculum e mysterium fidei. La chiesa cattolica in regime di lockdown
}

\author{
VINCENZO BOVA ${ }^{1}$
}

\begin{abstract}
Riassunto. Le conseguenze della pandemia sulla presenza della chiesa cattolica italiana. Il rapporto Stato-Chiesa. Le strategie di adattamento dei fedeli e dell'istituzione religiosa. Il ruolo di papa Francesco. Il cattolicesimo fra visibilità ed evanescenza. Le domande che si pongono all'istituzione e gli esiti possibili nel futuro modo di credere.
\end{abstract}

Parole chiave: pandemia, religione, cattolicesimo, rapporto Stato-Chiesa, lockdown.

\begin{abstract}
The consequences of the pandemic on the presence of the Catholic Church. The relation State-Church. The adaptive strategies by the church followers and by the religious institution. The role played by Pope Francis. The Catholicism between visibility and evanescence. The questions asked to the institution and the possible outcomes for the future way of believing.
\end{abstract}

Keywords: pandemic, religion, catholicism, State-Church relationship, lockdown.

\section{Introduzione}

Lesplosione della pandemia da Coronavirus rappresenta un evento inatteso che segna la nostra epoca definendo una linea di separazione fra il prima ed il dopo l'apparire dell'agente patogeno. Uno spettro si aggira per il mondo. Invisibile e potente. Inatteso, imprevisto ed i cui esiti non sono al momento calcolabili. Uno spettro che s'infiltra nella quotidianità di uomini e donne senza fare distinzioni di genere, nazionalità, ceto. Che attecchisce indipendentemente dai sistemi economici, dai sistemi politici, dalle culture. Corollario e compimento indesiderato di una tarda modernità che, dopo il crollo del muro di Berlino, tentava di riproporre la sua promessa primigenia: «domani sarà meglio di oggi» sotto il faro illuminante del processo di globalizzazione e delle potenze che ne sono a guardia. L'epidemia ha mostrato, in poche settimane, come il gigante cinese abbia piedi di argilla e come la sua fragilità infetti pericolosamente la rete di relazioni che aveva intessuto, a modo suo obbediente al percorso d'ingresso nel novero dei potenti della terra. Se nella società globalizzata «un battito d'ali di una farfalla ad est può provocare uno spaventoso uragano ad ovest» l'infezione partita dalla Cina ha mantenuto la stessa dinamica, con la forza originaria ben più temibile di quella esercitata da un battito d’ali di farfalla (qui il battito era di ali di pipistrello).

\footnotetext{
${ }^{1}$ Professore Ordinario di Sociologia dei processi culturali e comunicativi, Dipartimento di Scienze Politiche e Sociali, Università della Calabria. Email: vincenzo_antonino.bova@unical.it
} 


\section{Lo scenario italiano}

La diffusione del nuovo Coronavirus in Italia ha trovato, inizialmente, due prevalenti forme di lettura. La prima minimalista: è poco più di una banale influenza. La seconda catastrofista: è la nuova peste e siamo disarmati. A seconda della lettura, inizialmente si profilavano, con andamento altalenante, le decisioni di quanti avevano la responsabilità di direzione della vita pubblica. Il nostro Paese è sicuro, chiudiamo le frontiere alla Cina, il nostro sistema sanitario è eccellente. Poi in pochi giorni il crollo e ci scopriamo la prima nazione al mondo per numero di infettati. I toni cambiano, l'angoscia cresce sull'humus delle incertezze, le parole assumono i toni della chiamata alle armi: «l'Italia è più forte del virus! », "Andrà tutto bene! ». La politica mette in seconda linea decisioni strategiche che possano venire dal suo interno e si consegna obbediente alle indicazioni degli scienziati. Anche queste, inizialmente, non proprio univoche. Il Covid-19 in poche settimane mette in crisi il nostro essere al mondo e del mondo. L'impotenza e la fragilità, che non abitano la mentalità dell'uomo contemporaneo, diventano la drammatica evidenza di cui si discute nei bar, nei luoghi di lavoro, nei social. A dire il vero il primo tentativo è quello di allontanare il senso di caducità dell'esistenza umana relegando il rischio ad una minima parte di popolazione, anziani e malati, quelli insomma che con l'idea che la vita possa finire ci fanno quotidianamente i conti. Per gli altri non cè problema e la paura è esorcizzata dal naturale vigore e dalla fiducia nella scienza. Allora soprattutto da parte di quelle fasce di popolazione che, a torto o a ragione, si sentivano ancora tutelate dalla corazza dell'invincibilità, i più giovani, si attuavano comportamenti imprudenti che favorivano la diffusione del virus. Poi, man mano che l'epidemia si mostrava nella sua potenza espansiva e nei suoi effetti più letali, arrivano le drastiche decisioni che, partendo dalla delimitazione dei primi focolai di infezione, arrivano in poche settimane ad estendersi all'intero territorio nazionale. Il Covid-19 disegna i confini di comunità forti da cui, per decreto, provvisoriamente non si può uscire ed in cui non si può entrare (Diamanti, 2020). Non cè libertà di scelta. Si è costretti ad essere "parte di». Parte di uno spazio abitato dipinto di rosso o di giallo ed i cui confini sono decisi da chi tiene il conto dei livelli di diffusione del contagio. Altro che società liquida. Il Covid-19 può essere, se al momento non sconfitto, almeno contenuto nei suoi più nefasti effetti. Basta rispettare le regole: lavarsi spesso le mani, niente baci, niente abbracci e distanza di sicurezza tra l'uno e l'altro (un metro pare sufficiente), rimanere a casa e ridurre al minimo le relazioni sociali. Spazi di vita comunitari chiusi (scuole, università, chiese, palestre, bar, negozi).

Come nello stato di guerra la quotidianità di tutti appare sconvolta. Come in guerra, quando si è sotto attacco di un potente nemico bisogna mettere da parte le divisioni, bisogna essere uniti e ciascuno è chiamato a svolgere responsabilmente il proprio compito. La mia vita dipende dal tuo comportamento, la tua vita dipende dal mio. Per una volta il comportamento del singolo non è considerato secondo i criteri della marginalità degli effetti della sua azione. Al contrario l'azione del singolo, come nei comportamenti più irrazionali della folla, a suo tempo descritti da Le Bon (2014), diventa contagiosa ed è responsabile della diffusione di buone o nefaste pratiche. La legge dei rendimenti marginali decrescenti non trova qui applicazione, ogni comportamento virtuoso sommandosi ai precedenti non porta alla progressiva diminuzione dei benefici, ma li accresce in maniera esponenziale. E lo stesso, ovviamente accade quando il singolo comportamento si muo- 
ve nella direzione opposta. Come in una guerra di fronte ad un nemico la cui avanzata appare inarrestabile, la strategia che viene richiesta è quella di indietreggiare, lasciandogli davanti terra bruciata, allontanandolo dalle sue centrali di approvvigionamento, lasciandolo esposto ad ambienti ostici per la sua sopravvivenza, per avere il tempo di contrattaccare quando il nemico sarà indebolito e noi meglio organizzati.

I provvedimenti, imposti dal governo e dalle altre autorità competenti, per contrastare la diffusione del virus pongono dei limiti alla libertà dei cittadini. La parola chiave è «distanziamento sociale». Limitare allo stretto indispensabile i rapporti sociali. Lo slogan è «io sto a casa». Le mura di casa, le convivenze forzate assumono i connotati dell'istituzione totale, la cui evasione è in parte consentita dall'uso dei social o dalla concessione di permessi d'uscita per comprovate esigenze riconosciute come legittime dalle autorità.

Nell'attrezzarci insieme per combattere l'invisibile nemico siamo chiamati a fare i conti con gli effetti collaterali di un attacco che non mina solo alla nostra salute fisica, ma che ci interroga sul senso più vero della vita e della relazione con il mondo che ci circonda.

Scopriamo quanto il rapporto con gli altri è essenziale per il nostro benessere. Ma scopriamo anche che non sappiamo stare a casa e cosa fare stando a casa quando la presenza dell'altro (marito, moglie, figlio) eccede i tempi che di solito sono lasciati liberi dal lavoro per il mercato e dal consumo. Chi è l'altro da me quando è tirato fuori dalla routine cui eravamo abituati? Di fronte al grande nemico ci sentiamo tutti uniti, una sensazione di comunità, di qualcosa di creduto perso e che emerge come sensazione assopita. La solidarietà, la responsabilità di ciascuno nei confronti dell'altro ha una dinamica che non spinge alla vicinanza, ma tanto più si è solidali e responsabili quanto più si è capaci di mantenere una distanza fisica dall'altro. Esperienze nuove su cui non abbiamo una tradizione di comportamento.

L'altro da cui star lontano (potrebbe infettarmi), l'altro che rischia la sua vita per prendersi cura di me (gli operatori del servizio sanitario). Laltro da cui guardarsi, l'altro che ti soccorre. Diffidenti dal primo, grati al secondo. Come in guerra è sollecitato forte il richiamo all'unità, al sentirci un noi. Affacciati ai balconi, a dirci l'un l'altro che ce la faremo, orgogliosi di una comune identità nazionale, questa volta esibita senza distinguo. I mezzi tecnologici della comunicazione vengono sdoganati dallo stigma di alienazione ed esaltati come irrinunciabile risorsa per farci sentire vivi e partecipi di quel mondo che siamo stati costretti a lasciare fuori dalla porta di casa. Un distanziamento sociale imposto, obbedito e che sembra generare vicinanza. Immersi in un comune destino il cui esito, per una volta, pare affidato alla responsabilità del singolo. Con un notabene per uscire dall'alveo di un ingenuo sentimentalismo. L'ipertrofia dell'io sa come navigare traendo il massimo possibile dei vantaggi per sé dalla relazione con gli altri. Il noi che nasce dalla paura è transitorio e strumentale, per certi versi è l'uso reciproco dell'altro guidato da una logica fissata sull'io. Chi era per noi ieri il nostro dirimpettaio? Chi è per noi oggi, quando ci affacciamo al balcone per dire al mondo quanto il nostro popolo sia tenace? Chi sarà per noi domani, quando la paura sarà finita? La pandemia ha sparigliato le ingenue certezze della società contemporanea. Un atto di reciproca fiducia, non emozionale, ma generato dalla rivisitazione dei sistemi di interesse e di valore su cui ci costituiamo come nazione è la sola opportunità che ci è data, perché la tragedia che stiamo vivendo non si chiuda soltanto con la conta dei fortunati che ce l'hanno fatta e dei più deboli che sono rimasti sul campo. 


\section{La chiesa cattolica}

La pandemia ha rimesso in discussione il nostro stile di vita, i nostri tempi, i nostri spazi, il nostro modo di produrre e di consumare. Le parole di Ilaria Capua, virologa, direttrice dell'Emerging Pathogens Institute dell'Università della Florida danno la dimensione dell'evento: «Si poteva arrivare più preparati ma sfido chiunque a dire che tutto questo poteva essere previsto nella sua estensione. Stiamo vivendo un grandissimo esperimento evolutivo (...) Non cè dubbio che di tutto questo conserveremo i segni più nella coscienza che nei corpi» (Ventura, 2020).

Le questioni sin qui richiamate interrogano le istituzioni e, come in ogni momento rivoluzionario, ne provano l'adeguatezza nell'attraversare il momento specifico e nel riproporsi quando dallo stato emergenziale, dalla fiammata si tornerà, trasformati, ad una nuova «normalità». Questo contributo vuole riflettere sul modo con cui la Chiesa cattolica si è rapportata alle mutate situazioni di contesto indotte dalla diffusione in Italia della pandemia. Sono molteplici le questioni meritorie di riflessione di fronte ad una serie di accadimenti che hanno interrogato la Chiesa cattolica sia in quanto istituzione, sia in quanto comunità di fedeli. Un'istituzione che occupa un posto di rilievo nel nostro Paese e che mantiene una familiarità accentuata con le tematiche prima richiamate: senso della vita, fragilità dell'esistenza, morte, solidarietà, obbedienza, identità nazionale.

Il senso religioso trova uno spazio di rilievo nelle costruzioni sociali che formano le comunità umane. Esso si pone sotto forma di domande che in quanto uomini ed in quanto esseri sociali ci poniamo. Forse nessuno ha reso palpabili queste dinamiche meglio di come abbia fatto Leopardi attraverso i quesiti che accompagnavano la notte del suo pastore errante: perché c’è il dolore? Che senso ha la vita? Perché nonostante tutto continuiamo a fare figli? Che c'entro io con il creato? E soprattutto: io chi sono? Di fronte a interrogativi come quelli appena richiamati, l'esperienza religiosa si propone come una risposta che il credente ritiene adeguata a fare intravedere un percorso che traduce in sensatezza ciò che appare insensato, in ordine ciò che appare casuale, in compagnia ciò che è solitudine, in sicurezza ciò che è rischio. Nel contempo la relazione con l'oggetto della credenza non ha esclusiva funzione di conforto e stabilizzazione della psiche individuale, ma crea forme di relazionalità sociale legittimate dalla condivisione della credenza: religa i tanti diversi in una realtà collettiva che li rende simili. La religione crea legami, detta regole, legittima o delegittima il potere, crea nuovi modi di produzione ${ }^{2}$. La religione è percorso di «salvezza» individuale ma è anche percorso di formazione di identità collettive. Le religioni rimandano ad una trascendenza ma le religioni rispondono a bisogni dell'aldiquà. La religione non si rivolge a spiegare l'eccezionale o l'extra-ordinario, ma ciò che è "costante e regolare» (Durkheim, 1973, p. 42). Weber (1999) scriveva che: «Lagire religiosamente o magicamente motivato trae la sua originaria consistenza da un processo mondano. Le azioni che si presentano come religiose o magiche debbono venir compiute "affinché tutto ti vada bene e tu viva a lungo sulla terra"» (p. 105). Nella frase del sociologo tedesco, l'agire religioso ha come suo fondamento non il tentativo o il desiderio di sfuggire alla dimensione quotidiana della vita, ma piuttosto quello di trovare, attraverso la religione, una risorsa che consenta

\footnotetext{
${ }^{2}$ Se per Durkheim la scoperta della religione fu una «rivelazione» che lo costrinse «a ripensare tutte le precedenti ricerche» non molto diversamente si può dire di Comte, Weber e dello stesso Marx. Tutti, sia pur da prospettive diverse, incontrano e prestano attenzione a questo oggetto.
} 
di viver meglio nel quotidiano. Anche nella società secolarizzata, losservazione sociologica non può fare a meno di notare due cose: la persistenza del fenomeno religioso e la difficoltà di trovare un succedaneo di questa risorsa di coesione sociale (Casanova, 2000; Taylor, 2009). Anche nella società contemporanea, in quel mondo «di incertezza religiosa» (Berger, 1987, p. 61), in cui «la religione non legittima più "il mondo"» (Berger 1984, p. 165), in cui si infrange la catena della memoria (Hervieu-Léger, 2003) e la religiosità e le istituzioni in cui si incarna, diventano oggetto di scelta individuale e le chiese sono in competizione tra di loro e con altri concorrenti, che tendono ad erodere gli spazi e le domande a cui abitualmente rispondevano le chiese (basti pensare allo sviluppo della psicanalisi o alle più recenti pratiche del new age), la religione continua a esistere. Non sparisce, si adatta, si differenzia, si pluralizza, si mimetizza ma continua per tanti ad essere una risorsa dotata di senso ${ }^{3}$. La razionalità dell'uomo religioso è in un percorso di verifica di «convenienza» ad interpretare la sua esistenza con i parametri dettati da una credenza. Fintantoché il credere aiuta a vivere meglio è ragionevole continuare a farlo. Se qualcosa cè stata e continua ad esserci, è fuor di dubbio che ciò dipenda dal fatto che essa svolga una funzione sociale, una funzione che non trova altri e più adeguati strumenti che la sostituiscano. Come è stato osservato: «La religione della società non è senza relazione con la sincerità ed il fervore della fede personale: se non ci fossero più credenti, non esisterebbe più il problema del rapporto fra società e religione» (Rémond, 2003, p. 7-8).

La Chiesa cattolica «esperta in umanità» (Paolo VI, 1967) ancora oggi occupa un posto di tutto rilievo nella vita di tanti italiani in virtù delle funzioni e dei servizi da essa svolti. "In una società che accentua il suo carattere multietnico e multireligioso, una quota rilevante di popolazione sembra ritrovare nella fede della tradizione una risorsa simbolica capace di offrire sicurezza nella nuova situazione (...) Nella società dell'incertezza molti individui possono così riscoprire nell'appartenenza cattolica un sentire comune in grado di alimentare il legame sociale (...) Si tratta di una forma di adesione al cattolicesimo che non impegna necessariamente chi la esprime né alla pratica religiosa, né all'accettazione delle norme della chiesa» (Garelli, 2020, p. 57).

\section{Stato e chiesa cattolica di fronte all'emergenza}

I provvedimenti ${ }^{4}$ emanati dalle autorità politiche per la gestione dell'emergenza Covid-19 hanno, ovviamente, interessato la Chiesa cattolica e la sua modalità di presenza sia in quanto istituzione sia in quanto insieme di cittadini che, in maniera più o meno attiva e più o meno fedele, trovano nella Chiesa orientamenti, risposte e spazi di socialità ritenuti utili per attraversare le vicende dell'umana esistenza ${ }^{5}$. Ci troviamo di fronte

\footnotetext{
${ }^{3}$ Sulla permanenza della dimensione religiosa al centro della modernità come processo culturale rimando a Abbruzzese, 2010; Berzano, 2014; Bova, 2014; Berger, 2017.

${ }^{4}$ Il Decreto del Presidente del Consiglio dei Ministri dell'8 marzo 2020 così recitava: «l'apertura dei luoghi di culto è condizionata all'adozione di misure organizzative tali da evitare assembramenti di persone, tenendo conto delle dimensioni e delle caratteristiche dei luoghi, e tali da garantire ai frequentatori la possibilità di rispettare la distanza tra loro di almeno un metro (...) Sono sospese le cerimonie civili e religiose, ivi comprese quelle funebri».

${ }^{5}$ Una rassegna delle modalità di ridefinizione di tempi e modalità del culto delle istituzioni religiose non cattoliche è documentata dai contributi pubblicati nel sito dell'OLIR -Osservatorio delle libertà e delle istituzioni religiose, www.olir.it
} 
ad un evento di particolare rilevanza. Un provvedimento dello Stato italiano che limita in maniera importante la vita ordinaria dei cattolici. Una questione che ha a che vedere col rapporto Stato-Chiesa e con l'obbedienza al principio di autorità. La Conferenza Episcopale Italiana risponde al Dpcm in maniera coerente ai contenuti della Dottrina sociale cattolica: «L'autonomia reciproca della Chiesa e della comunità politica non comporta una separazione che escluda la loro collaborazione: entrambe, anche se a titolo diverso, sono al servizio della vocazione personale e sociale dei medesimi uomini. La Chiesa e la comunità politica, infatti, si esprimono in forme organizzative che non sono fini a se stesse, ma al servizio dell'uomo, per consentirgli il pieno esercizio dei suoi diritti, inerenti alla sua identità di cittadino e di cristiano, e un corretto adempimento dei corrispondenti doveri. La Chiesa e la comunità politica possono svolgere il loro servizio "a vantaggio di tutti in maniera tanto più efficace quanto meglio entrambe allacciano tra loro una sana collaborazione, considerando anche le circostanze di luogo e di tempo"» (Pontificio consiglio della giustizia e della pace, 2004, p. 425). Il giorno stesso dell'entrata in vigore del Dpcm la CEI, che fino a quel momento aveva stabilito delle norme di prudenza meno stringenti (svuotare le acquasantiere, non dare il segno della pace, dare la particola nelle mani e non in bocca), aderisce alle richieste del governo con un comunicato di poche essenziali righe: «La Chiesa che vive in Italia e, attraverso le Diocesi e le parrocchie si rende prossima a ogni uomo, condivide la comune preoccupazione, di fronte all'emergenza sanitaria che sta interessando il Paese. Rispetto a tale situazione, la CEI - all'interno di un rapporto di confronto e di collaborazione - in queste settimane ha fatto proprie, rilanciandole, le misure attraverso le quali il Governo è impegnato a contrastare la diffusione del "coronavirus". Il Decreto della Presidenza del Consiglio dei Ministri, entrato in vigore questoggi, sospende a livello preventivo, fino a venerdì 3 aprile, sull'intero territorio nazionale "le cerimonie civili e religiose, ivi comprese quelle funebri". L'interpretazione fornita dal Governo include rigorosamente le Sante Messe e le esequie tra le "cerimonie religiose". Si tratta di un passaggio fortemente restrittivo, la cui accoglienza incontra sofferenze e difficoltà nei Pastori, nei sacerdoti e nei fedeli. Laccoglienza del Decreto è mediata unicamente dalla volontà di fare, anche in questo frangente, la propria parte per contribuire alla tutela della salute pubblica» (Conferenza episcopale italiana, 2020).

Si chiudono le celebrazioni e le altre attività ospitate nelle parrocchie. Leffetto del decreto governativo, combinato con le disposizioni date dai vescovi, portano anche alla sospensione delle attività svolte in compresenza dalle aggregazioni laicali. Così l'Azione Cattolica: «L'emergenza sanitaria legata al Coronavirus sta colpendo tutta l'Italia. Anche la vita dell'Azione Cattolica risente della situazione che stiamo attraversando. A tutti i soci e agli amici dell'Azione Cattolica vorremmo dire che la vita associativa non si è fermata e non si fermerà. Certo, non possiamo portare avanti le attività ordinarie nel modo in cui siamo abituati. Tuttavia, l'associazione è presente con creatività e dedizione e le tante esperienze attivate in questi giorni ce ne danno conferma»(Presidenza e Consiglio nazionale Ac, 2020). Così l'Agesci: «Le disposizioni arrivate ieri sera sono chiare nell'invito di limitare al minimo gli spostamenti, salvo necessità reali e ben specificate. E su queste disposizioni noi dobbiamo mettere in campo tutta la nostra fedeltà nell'essere cittadini responsabili, sentendoci ancor di più fratelli l'uno dell'altro. Vi chiediamo particolare attenzione in tutte quelle realtà dove sappiamo che singoli Gruppi, per mettersi al passo degli ultimi, attivano iniziative di servizio che, se estemporanee e senza nessun coordinamento, potreb- 
bero mettere a repentaglio la salute di capi e ragazzi e di tutti coloro che sono beneficiari del prezioso servizio e di chi sta loro vicino. Questo non è possibile» (Agesci, 2020). Così Comunione e liberazione: «Lattuale emergenza sanitaria e le problematiche legate allorganizzazione dei nostri gesti ci impongono di disdire tutti i consueti appuntamenti di questo momento dell'anno... Questa decisione, imposta dall'emergenza, non fa sparire la presenza insidiosa del coronavirus tra di noi né attenua la provocazione che essa rappresenta, non ci consente di voltare la faccia dall'altra parte, come se non ci toccasse. Volenti o nolenti, ci riguarda tutti. E, con tutti, noi condividiamo la stessa domanda: come stare da uomini davanti a questa circostanza?» (Carron, 2020). Così le Acli: «L’emergenza Coronavirus mette a dura prova il Paese e ormai il mondo intero. Contenere il contagio e far sì che si possa assistere al meglio chi ne è colpito sono le priorità. Per questo occorre innanzitutto stringersi attorno alle nostre istituzioni e collaborare, ognuno restando nel proprio ruolo e nelle proprie competenze, perché si possa lottare al meglio. Noi Acli con i nostri circoli, associazioni e servizi ci siamo da tempo come presidio nelle e delle comunità» (Direzione nazionale Acli, 2020). Così Rinnovamento nello Spirito Santo: «Non è stato semplice accogliere le disposizioni adottate. Il bene grande della libertà religiosa e di culto è stato messo a dura prova, al pari di tutte le altre libertà individuali, che consideravamo conquiste acquisite una volta per tutte. Solo quando l'aria manca e ci sembra di soffocare, allora ne recuperiamo tutto il valore vitale!» (Gnagni, 2020). E il movimento inserisce «\#iorestoacasa» in basso al centro del proprio logo accompagnato nel resto della circonferenza dalle parole che delineano i contenuti dello stare a casa: intercedo, prego, dono. Così il Movimento dei Focolari che: « invita la grande famiglia dei Focolari nel mondo a vivere con la necessaria attenzione e con grande senso di responsabilità questa emergenza sanitaria in vista della salute personale e del bene comune. In particolare il Movimento dei Focolari raccomanda di adottare in modo scrupoloso le misure di precauzione e sicurezza stabilite dalle autorità sanitarie del proprio Paese e seguire con attenzione le relative comunicazioni» (Movimento dei Focolari, 2020).

Vita associativa che continua, seppur con modalità differenti. Opere ecclesiali di servizio e assistenza che riorganizzano la loro azione sul territorio nazionale. Ma quanto questa pausa inciderà su quel livello di prossimità fisica, nell'essere e nel fare assieme, che sostanzia la dimensione comunitaria delle aggregazioni cattoliche?

\section{Cittadini e credenti a navigare nella doppia fedeltà}

Ladozione da parte delle Cei delle misure stabilite dal Governo, genera una serie di perplessità trasversalmente espresse sia fra i vertici che nella base di una parte del variegato mondo cattolico. Papa Francesco, il Papa della «chiesa in uscita» e della «chiesa ospedale da campo» interviene direttamente sulle direttive emanate dalla Diocesi di Roma e chiede di riaprire le chiese. A distanza di poche ore dallo scarno comunicato di chiusura delle celebrazioni religiose, la Cei ritorna sull'argomento a specificare che il provvedimento restrittivo non intende chiudere la presenza della Chiesa accanto ai suoi fedeli, presenza che continua, seppur mutilata delle celebrazioni collettive, privazione resa necessaria dall'agire nella direzione della difesa del bene comune: «È una Chiesa, la nostra, presente, anche in questo frangente, nella carità: siamo edificati da tanti volontari delle Caritas, delle parrocchie, dei gruppi, delle associazioni giovanili, delle Misericordie, delle Confra- 
ternite $(. .$.$) che si adoperano per sollevare e aiutare i più fragili. "I cristiani non si diffe-$ renziano dagli altri uomini - osserva la lettera A Diogneto -: vivono nella carne, ma non secondo la carne. Vivono sulla terra, ma hanno la loro cittadinanza in cielo". È con questo sguardo di fiducia, speranza e carità che intendiamo affrontare questa stagione. Ne è parte anche la condivisione delle limitazioni a cui ogni cittadino è sottoposto. A ciascuno, in particolare, viene chiesto di avere la massima attenzione, perché un'eventuale sua imprudenza nellosservare le misure sanitarie potrebbe danneggiare altre persone. Di questa responsabilità può essere espressione anche la decisione di chiudere le chiese. Questo non perché lo Stato ce lo imponga, ma per un senso di appartenenza alla famiglia umana, esposta a un virus di cui ancora non conosciamo la natura né la propagazione» (Conferenza Episcopale Italiana, 2020a). Pochi giorni dopo, un documento di vicinanza al Papa ed alla posizione espressa dai Vescovi, viene sottoscritto e reso pubblico da 38 associazioni cattoliche e da 17 parlamentari o ex parlamentari cattolici (Ognibene, 2020).

Insomma, non poteva essere diversamente, di fronte ad un evento che mai si era presentato nella storia della Chiesa cattolica italiana le perplessità non nascono solo dall'anziana signora privata del quotidiano conforto della partecipazione liturgica, ma vengono poste da più parti con toni talvolta perentori, altre volte attenuati dal succedersi rapido di informazioni sull'inattesa potenza letale della pandemia.

Nei fatti, come ha sottolineato Massimo Franco (2020): «si capisce che il problema non è una contrapposizione tra Chiesa italiana e governo, in un momento in cui deve essere massima la coesione nazionale. Le obiezioni che filtrano riguardano l'esigenza dello Stato di dettare regole per la sicurezza e la salute, compresa l'eventuale chiusura delle chiese; ma in parallelo il diritto-dovere delle autorità religiose di attenersi a queste regole, decidendo autonomamente come gestire le cerimonie religiose senza violare le limitazioni (...) Il dilemma scopre in realtà il nervo della crescente irrilevanza della presenza cattolica nel Paese; e la debolezza di una Chiesa dai riflessi lenti, insieme intimidita e disorientata». Un giudizio tranchant cui si potrebbe obiettare che non solo la Chiesa ma anche le istituzioni laiche sono apparse disorientate e intimidite dall'inatteso esplodere della pandemia.

Tuttavia, una buona eco di questa interpretazione è in un'intervista, rilasciata da Andrea Riccardi (2020), fondatore della Comunità di Sant'Egidio, quando le misure di contenimento ancora non erano estese a tutta Italia, ma riguardavano solo le regioni del nord: «La prudenza serve, ma forse ci siamo fatti prendere la mano dalla grande protagonista del tempo: la "paura" (...) Le chiese non sono solo "assembramento" a rischio, ma anche un luogo dello spirito: una risorsa in tempi difficili, che suscita speranza, consola e ricorda che non ci si salva da soli (...) Nemmeno ai tempi dei bombardamenti e del passaggio del fronte durante la seconda guerra mondiale (quando la Chiesa fu l'anima della tenuta di un popolo), si chiudevano le chiese e si sospendevano le preghiere. Anzi il popolo si radunava fiducioso in esse, nonostante i pericoli di bombe e massacri. Forse la collaborazione dell'autorità ecclesiastica locale con quelle regionali è stata troppo intesa come subordinazione a quest'ultima. Si finisce così per banalizzare la presenza e l'apporto della Chiesa, che dà invece un suo contributo alla vita delle persone (...) Il "silenzio" e la solitudine religiosa sono un aggravio tra le difficoltà».

Quale spazio è riconosciuto alla Chiesa cattolica, in un momento in cui le grandi questioni, relative all'esistenza del bene e del male, dell'identità nazionale, della solidarietà, della morte, del sacrificio, del sostegno psicologico per contenere il disagio derivante 
dalle nuove condizioni di vita, dell'obbedienza alle autorità, dell'identità nazionale si pongono con forza all'ordine del giorno, nella ricerca di quella pluralità di risorse che consenta di superare il momento di crisi e ripartire? Se può rimanere aperta una fabbrica, un supermercato o una farmacia o un qualunque altro servizio ritenuto indispensabile, con le capacità che ciascuno di essi ha di porre in atto sistemi di sicurezza per quanti condividono una prossimità fisica, perché la stessa cosa non è pensabile per le cerimonie religiose? Nell'urlo di senso che accompagna la tragedia, le cerimonie religiose, la messa domenicale, il rito di commiato ai defunti sono una risorsa particolare da tenere in campo, o sono equiparabili ad altri rituali che scandiscono la nostra vita (palestre, discoteche, centri commerciali ecc.)? Ed ancor più la domanda si pone a fronte della constatazione che i soggetti più a rischio sono in larga parte anziani, cioè quella parte della popolazione maggiormente ancorata alla tradizionale fede di Chiesa. E da ultimo, quale spazio di autonomia è stato lasciato alla Chiesa, o la Chiesa ha difeso, a fronte di decisioni che ne limitavano gravemente l'agire? La Cei poteva disubbidire e cosa sarebbe in quel caso accaduto?

Marco Politi (2020), a proposito delle questione di cui stiamo trattando, arriva a parlare di evanescenza della religione dalla sfera pubblica: «Mai come in questa epidemia è apparsa così evidente l'eclissi della religione dalla scena pubblica. Per la prima volta dai tempi del medioevo un grande fenomeno come la peste imperversa e domina ogni spazio nell'assenza totale dei simboli religiosi. Prova più lampante della secolarizzazione e del suo spessore non poteva esserci (...) Nella civiltà dell'immagine l'assenza risalta. Sul palcoscenico odierno svanisce la Religione, resta padrona incontrastata la Scienza». Evanescenza in quell'Italia in cui: «la maggioranza degli italiani (56\%) continua ad apprezzare il ruolo della parrocchia nei diversi territori» (Garelli, 2020, p. 130) e "circa i due terzi degli italiani dichiarano che la religione permette loro di "comprendere il senso profondo della vita", mentre poco meno del $60 \%$ afferma di trovare a questo livello motivi per rasserenarsi di fronte alla morte» (Ibidem, p. 38). Un'Italia in cui, come si rileva da una indagine condotta dall'istituto demografico Ipsos, durante il periodo più acuto della pandemia, si assiste ad una intensificazione della vita religiosa: «un quarto degli italiani avverte oggi l'esigenza di una vita spirituale più intensa, e in parallelo sente Dio più prossimo alla propria condizione di vita. In questo scenario, vi è anche un incremento della preghiera, dichiarato dal $16 \%$ del campione. In tutti i casi, il termometro religioso del paese sembra rivolto all'insù; soprattutto per il maggior numero di persone più sensibili al lato spirituale dellesistenza e al rapporto con Dio rispetto a quanti sono spinti-anche da questa pandemia - a negare un riferimento trascendente o a non dar rilievo ai valori dello spirito» (Garelli, 2020a). Evanescenza di fronte ad un tema che, in tanti, rimanda alla questione della teodicea, dell'esistenza del bene e del male e dell'azione di un Dio presente e che opera nella storia: «Il Coronavirus, come le epidemie del passato, i terremoti o gli tsunami, suscita l'interrogativo sul senso del male fisico, quello non causato dalla cattiveria del malvagio, ma dai processi della natura, di cui siamo parte. Non essendovi un nemico da disarmare, è Dio che finisce presto sul banco degli imputati» (Tanzella-Nitti, 2020).

Non intendo approfondire le questioni che sono state appena poste, se non alla luce delle riflessioni che Olivier Roy (2019) pone sulle dinamiche del processo di secolarizzazione derivanti dalla capitolazione delle chiese nei confronti dall'autorità statuale. Stato a cui sempre più le chiese si rivolgono fiduciose per assumere decisioni inerenti le specifiche sfere di azione della religione. Chiedono allo Stato ed al suo apparato giudiziario di sta- 
bilire e tutelare comportamenti la cui tutela era precedentemente materia di monopolio esercitato dall'autorità religiosa, riconoscendo così la propria incapacità a difenderli per autorità propria. Offerte religiose fragili, afone, frammentate che trovano convenienza ad avere un difensore di quegli spazi che le vengono garantiti, in una competizione regolamentata dallo Stato. Chiese che riducono l'offerta spirituale omologandosi alla domanda proveniente da una società secolarizzata.

\section{Nel mare in tempesta. La Barca di Pietro e le scialuppe di salvataggio}

La seconda questione su cui intendo soffermarmi è invece interna alla vita della Chiesa cattolica. Quali conseguenze potrà avere la sospensione di pratiche religiose pubbliche nel modo in cui gli italiani vivono la loro esperienza di fede ed il loro rapporto con la Chiesa cattolica? In che modo inciderà la sospensione di determinate pratiche (messa, comunione, confessione, unzione degli infermi, intermediazione del clero, associazionismo) espressione comunitaria e personale della fede, soprattutto in quelle nuove generazioni che saranno particolarmente segnate dall'evento storico che stiamo attraversando, se non altro perché avranno più tempo in cui conservarlo nella memoria? È difficile prevederlo ma al momento è condivisibile quanto osserva Berzano (2020): «nel tempo delle chiese chiuse per il virus, sono nate nel mondo cattolico sperimentazioni liturgiche con celebrazioni, incontri e preghiere online nelle quali rituali sacri millenari sono stati privati della loro essenza materiale e interattiva con i fedeli, per diventare video e programmi da vedere. Tutto è stato coinvolto in una generale dematerializzazione (...) la religione perde così la sua realtà corporea, divenendo uno "spettacolo" da vedere. Il mondo religioso diventa immateriale, le celebrazioni liturgiche si trasformano in immagini e tutto diventa realtà digitale, cioè simulacro (...) la religione, concepita in senso immateriale, non è più esperienza costruita, ma solo consumata, per la sua carica simbolica, spettacolare, e per il suo valore emozionale. Tutto appartiene alla dimensione spettacolare, che invade non solo la realtà economica e sociale, ma anche la vita interiore, le aspirazioni, i sogni». Non è superfluo precisare inoltre che, come recita il Catechismo della Chiesa Cattolica al punto 1069: "Il termine "liturgia" significa originalmente "opera pubblica", "servizio da parte del popolo e in favore del popolo". Nella tradizione cristiana vuole significare che il popolo di Dio partecipa all opera di Dio. Attraverso la liturgia Cristo, nostro Redentore e Sommo Sacerdote, continua nella sua Chiesa, con essa e per mezzo di essa, l'opera della nostra redenzione». In termini semplici non c’è liturgia se non c’è popolo.

L'impossibilità da parte dei fedeli di accedere comunitariamente ai luoghi di culto, l'impossibilità di ricevere la comunione, aprono uno scenario che estende all'intera comunità dei credenti le speciali condizioni che erano previste per quanti fossero impediti a partecipare alla celebrazione eucaristica. Coloro che per condizioni di salute, per età, per doveri di assistenza verso altri non poteva recarsi in chiesa erano esonerati da questo obbligo e potevano seguire la funzione attraverso la radio o la televisione. Nella vita ordinaria della Chiesa cattolica quanti erano in condizione di fragilità potevano, quindi, seguire da casa la santa messa e ricevere lostia consacrata portata nelle loro case da sacerdoti, diaconi o ministri straordinari. Laccettazione, da parte della Cei, delle misure del Governo, estende a tutti la condizione di fragilità (la pandemia ci rende tutti potenzialmente malati) e contrae sia la dimensione orizzontale (incontro fra credenti) che quella verticale (incontro fra cre- 
dente e oggetto della credenza). In queste condizioni, la dimensione religiosa subisce da un lato una spinta alla privatizzazione (la famiglia come chiesa domestica) dall'altro una spinta all'espulsione della trascendenza dalla vita quotidiana. La messa si può seguire da casa, in tv o sui social, per pregare non cè bisogno di andare in chiesa, il corpo e il sangue di Cristo sono sostituiti dalla comunione spirituale. Una momentanea deogettivizzazione dei luoghi del sacro e di incremento dei livelli di autonomia del credente nell'interpretazione di un percorso di salvezza. L'eucarestia è centro, sostanza e significato della celebrazione domenicale. Per i credenti è l'incontro con la presenza fisica della dimensione trascendente. Un significato quindi non marginale della vita religiosa. Leucaristia è un sacramento e per la Chiesa cattolica «I sacramenti sono segni efficaci della grazia, istituiti da Cristo e affidati alla Chiesa, attraverso i quali ci viene elargita la vita divina». Tutto questo avendo come riferimento un'Italia in cui, ancora oggi, il 22\% della popolazione va regolarmente a messa, un altro $15 \%$ sale i gradini del sagrato almeno una o due volte al mese ed il $60 \%$ di quelli che partecipano al rito fa la comunione (Garelli, 2020).

Se è fuor di dubbio che ridotta sia la quota di chi possa pensare che la pandemia sia stata scatenata da un Dio che voglia vendicarsi dell'infedeltà del genere umano ${ }^{6}$ e che bisogna fare qualcosa per convincerlo ad avere misericordia, è anche fuor di dubbio che la credenza assai più diffusa, con livelli assai prossimi alla certezza, è che sarà la scienza a liberarci dal male e che onori, sacrifici e gloria vanno in questo momento tributati a quanti sono in prima linea per raggiungere questo obiettivo (medici, infermieri, protezione civile, volontariato ecc). Se forte in tutto il Paese è il richiamo al sacrificio (a rischio spesso della stessa vita) di quanti sono chiamati a svolgere i cosiddetti servizi essenziali perché, almeno inizialmente, tenue è apparsa la resistenza volta a difendere come essenziale anche il servizio religioso? Al sacrificio (sacrum facere) non potevano essere chiamati anche coloro che per vocazione si occupano della cura dello spirito? Quanto peserà nella memoria collettiva del paese l'immagine di una chiesa «ospedale da campo» che sembra accettare di operare nelle retrovie, che si muove non di moto e strategia propria ma in modo subalterno alle indicazioni che vengono dal potere politico e dal mondo della scienza? Quanto peserà lo strazio dell'ultima drammatica fase di passaggio, la morte, che non può essere accompagnata dal conforto di un sacerdote, di una salma che non può essere onorata dal rito dellestremo saluto? Ma poteva la Chiesa agire diversamente da come ha fatto? Scienza e prudenza suggeriscono di no.

Quanto osservato per l'eucarestia è riferibile anche ad un altro sacramento fondamentale della vita cristiana, la confessione. Anche la riconciliazione, la remissione dei peccati si struttura alla luce delle condizioni di eccezionalità del momento. Un decreto della Penitenzieria Apostolica del 19 marzo 2020, ex auctoritate Summi Pontificis, concede l'indulgenza plenaria ai fedeli affetti da Coronavirus, agli operatori sanitari, ai familiari e a quanti, esponendosi al rischio di contagio, assistono i malati di Coronavirus ${ }^{7}$. L'indulgenza

\footnotetext{
${ }^{6}$ «Contrariamente a molti luoghi comuni, la grande maggioranza (il 70\%) non equipara il Coronavirus ad un fulmine divino che si abbatte su un mondo ritenuto ormai pagano e secolarizzato. Lidea del Dio punitivo non sembra trovare - nemmeno in questa circostanza eccezionale - grande credito nella società contemporanea, anche se continua a far breccia in alcune minoranze religiose di umore apocalittico» (Garelli, 2020a).

${ }^{7}$ «Se, con l'animo distaccato da qualsiasi peccato, si uniranno spiritualmente attraverso i mezzi di comunicazione alla celebrazione della Santa Messa o della Divina Liturgia, alla recita del Santo Rosario o dell'Inno Akàthistos alla Madre di Dio, alla pia pratica della Via Crucis o dell'Ufficio della Paràklisis alla Madre di Dio oppure ad altre preghiere delle rispettive tradizioni orientali, ad altre forme di devozione, o se almeno reciteranno il Credo,
} 
plenaria è inoltre estesa ai fedeli che preghino per la cessazione dell'epidemia o per quanti ne soffrano le conseguenze ${ }^{8}$ e, da ultimo, per chi si trovasse nell'impossibilità di ricevere il sacramento dell'Unzione degli infermi e del Viatico 9.

Nel momento in cui il Paese vive i giorni più drammatici degli effetti della pandemia, la Chiesa assume misure straordinarie dirette ad assicurare il conforto dei sacramenti a quanti, con gradi diversi di compromissione, partecipano di questo particolare momento storico. Lo fa portando questo intervento non a beneficio di singoli o di categorie ma estendendolo in modo universale. Nell'ordinario, la sua presenza espulsa dalle corsie, al pari dei famigliari delle vittime dell'epidemia, si gioca negli altri spazi della vita sociale, con più di 100 sacerdoti che perdono la vita dopo avere contratto l'infezione da coronavirus.

\section{Dietro il sipario e sulla scena}

L'immagine di una Chiesa disorientata dal tragico turbinio degli eventi, messa all'angolo di uno scenario in cui altri e ben più visibili sono i protagonisti, si rovescia tuttavia in fretta. L'inizio è in un gesto di «disubbidienza». Il Papa disubbidiente, che nel pomeriggio della prima domenica di lockdown esce a piedi in una Roma deserta, per andare a pregare nella chiesa di San Marcello al Corso davanti al Crocifisso che nel 1522 venne fatto girare per 16 giorni per le vie della Capitale finché la peste non si interruppe. Francesco, con la sua catechesi dei gesti (Muolo, 2016), chiama ancora una volta i pastori a stare vicini al proprio gregge. Prossimità a quanti soffrono e preghiera. Se è superfluo riflettere sulla prima sollecitudine: la comunità cristiana ha sospeso le celebrazioni aperte ai fedeli, ma non la sua presenza attraverso una capillare rete di opere e di servizi sul territorio ${ }^{10}$, sulla seconda sollecitudine, il Santo Padre esorta ad una pandemia della preghiera, della tenerezza, della compassione.

Lappello del Papa trova un suo seguito. Il 19 marzo la Cei invita a sintonizzarsi su TV2000 alle 21 per partecipare ad una «Preghiera per l'Italia colpita dall'epidemia». La recita del rosario, che era parzialmente in contemporanea con la fortunata e seguitissima serie televisiva «Don Matteo», registra «qualcosa come cinque milioni e mezzo di uomini e donne in preghiera» (Politi 2020). La settimana successiva, il 27 marzo, 17 milioni di telespettatori partecipano ad un evento che segnerà la memoria collettiva. È l'immagine del vecchio e barcollante Papa che percorre, sotto la pioggia, i gradoni della Basilica di San Pietro,

\footnotetext{
il Padre Nostro e una pia invocazione alla Beata Vergine Maria, offrendo questa prova in spirito di fede in Dio e di carità verso i fratelli, con la volontà di adempiere le solite condizioni (confessione sacramentale, comunione eucaristica e preghiera secondo le intenzioni del Santo Padre), non appena sarà loro possibile» (Penitenzieria Apostolica, 2020).

8 «Fedeli che offrano la visita al Santissimo Sacramento, o l'adorazione eucaristica, o la lettura delle Sacre Scritture per almeno mezzora, o la recita del Santo Rosario o dell'Inno Akàthistos alla Madre di Dio, o il pio esercizio della Via Crucis, o la recita della Coroncina della Divina Misericordia, o dell'Ufficio della Paràklisis alla Madre di Dio o altre forme proprie delle rispettive tradizioni orientali di appartenenza per implorare da Dio Onnipotente la cessazione dell'epidemia, il sollievo per coloro che ne sono afflitti e la salvezza eterna di quanti il Signore ha chiamato a sé» (Ibidem)

9«Concede al fedele l'Indulgenza plenaria in punto di morte, purché sia debitamente disposto e abbia recitato abitualmente durante la vita qualche preghiera» Ibidem.

${ }^{10}$ Lapprezzamento per l'opera della Chiesa è esplicitamente formulato dal Presidente del Consiglio con una lettera aperta inviata al direttore del quotidiano della CEI. (Conte, 2020).
} 
in una piazza spettralmente vuota e prende ansimante la parola per rincuorare e chiamare all'unità un'umanità ferita, impaurita e disorientata: «Fitte tenebre si sono addensate sulle nostre piazze, strade e città; si sono impadronite delle nostre vite (...) Ci siamo trovati impauriti e smarriti. Come i discepoli del Vangelo siamo stati presi alla sprovvista da una tempesta inaspettata e furiosa. Ci siamo resi conto di trovarci sulla stessa barca, tutti fragili e disorientati, ma nello stesso tempo importanti e necessari, tutti chiamati a remare insieme» (Francesco, 2020). La preghiera di Francesco è un atto di critica verso un modo di vivere che genera una umanità vulnerabile ed indifesa: «La tempesta smaschera la nostra vulnerabilità e lascia scoperte quelle false e superflue sicurezze con cui abbiamo costruito le nostre agende, i nostri progetti, le nostre abitudini e priorità. Ci dimostra come abbiamo lasciato addormentato e abbandonato ciò che alimenta, sostiene e dà forza alla nostra vita e alla nostra comunità (...) Con la tempesta, è caduto il trucco di quegli stereotipi con cui mascheravamo i nostri "ego" sempre preoccupati della propria immagine; ed è rimasta scoperta, ancora una volta, quella (benedetta) appartenenza comune alla quale non possiamo sottrarci: l'appartenenza come fratelli»(Ibidem). Le parole del Pontefice sono l'invocazione del perdono per una umanità che ha eliminato dal suo orizzonte la dimensione della trascendenza, la storicità dell'incarnazione: «siamo andati avanti a tutta velocità, sentendoci forti e capaci in tutto. Avidi di guadagno, ci siamo lasciati assorbire dalle cose e frastornare dalla fretta. Non ci siamo fermati davanti ai tuoi richiami, non ci siamo ridestati di fronte a guerre e ingiustizie planetarie, non abbiamo ascoltato il grido dei poveri, e del nostro pianeta gravemente malato. Abbiamo proseguito imperterriti, pensando di rimanere sempre sani in un mondo malato» (Ibidem). Nelle parole di Francesco il richiamo ad una scelta di conversione: «Non è il tempo del Tuo giudizio, ma del nostro giudizio: il tempo di scegliere che cosa conta e che cosa passa, di separare ciò che è necessario da ciò che non lo è. È il tempo di reimpostare la rotta della vita verso di Te, Signore, e verso gli altri. E possiamo guardare a tanti compagni di viaggio esemplari, che, nella paura, hanno reagito donando la propria vita (...) medici, infermiere e infermieri, addetti dei supermercati, addetti alle pulizie, badanti, trasportatori, forze dell'ordine, volontari, sacerdoti, religiose e tanti ma tanti altri che hanno compreso che nessuno si salva da solo (...) La preghiera e il servizio silenzioso: sono le nostre armi vincenti» (Ibidem).

La religione non svanisce dalla scena pubblica. Se le funzioni religiose sono precluse ai fedeli, la partecipazione ai riti si moltiplica sui social così come lo spazio che i canali televisivi dedicano alle funzioni religiose. Al di là della visibilità data ai classici appuntamenti, si aggiungono i collegamenti per gli eventi straordinari e la rete televisiva pubblica Rail ogni mattina trasmette in diretta la messa celebrata da Papa Francesco nella cappella della residenza di Santa Marta e le sue omelie vengono regolarmente riprese nei servizi dei telegiornali.

Francesco guida la presenza della Chiesa dentro le cose del mondo. I suoi interventi accettano la sfida di dolore di quanti si chiedono dove sia Dio in questa circostanza, quel Dio padre buono che si prende cura dei suoi figli, ma le parole del Papa non hanno toni meramente consolatori o di miracolistiche attese dell'immediata fine del dramma collettivo che si sta vivendo. Nelle sue parole, nei suoi gesti, traspare visibile la prossimità alla sofferenza ${ }^{11}$ ma c’è anche la critica a quelle «strutture di peccato» (Giovanni Paolo II,

\footnotetext{
11 «da questo altare, da questo sacrificio di Gesù, di Gesù che non si è vergognato di piangere, chiediamo la grazia di piangere. Che oggi sia per tutti noi come la domenica del pianto» (Francesco, 2020a)
} 
1987) che segnano l'interdipendenza dei sistemi economici, politici, sociali. Il Papa della Laudato si di fronte all'esplosione della pandemia ha ben chiaro il ruolo che la scienza avrà nel superamento della crisi, ma ha anche chiaro che: «qualunque soluzione tecnica che le scienze pretendano di apportare sarà impotente a risolvere i gravi problemi del mondo se l'umanità perde la sua rotta, se si dimenticano le grandi motivazioni che rendono possibile il vivere insieme, il sacrificio, la bontà» (Francesco 2015, p. 200). Il gesto della benedizione Urbi et orbi, dell'indulgenza plenaria, dei gradoni saliti in solitudine, in prossimità alla solitudine dell'uomo morente, l'invocazione della misericordia di Dio, ma anche la richiesta forte di una conversione degli stili di vita individuali e dei paradigmi tecno economici che governano la vita collettiva ${ }^{12}$. Il papa argentino non si propone come la guida di una cittadella assediata dagli attacchi della secolarizzazione e bisognosa di legittimare la sua plausibilità dentro la tempesta epidemica. Il gesto di papa Bergoglio travalica i confini del mondo cattolico e della immediata contingenza. Leditoriale di un autorevole non credente, Eugenio Scalfari, pubblicato a commento dell'evento, pur se discutibile quanto a interpretazione autentica, testimonia della capacità di penetrazione del gesto posto dal Papa: «Francesco non è un mistico e per questa ragione ha preso quel nome che non gli somiglia affatto nella sostanza ma lo seduce estremamente. Lattitudine di questo Papa è la logica ed il desiderio di ricostruire la Chiesa aggiornandola alle caratteristiche della società moderna che comincia da Montaigne e dall'illuminismo di Voltaire e Diderot (...) Da questo punto di vista papa Bergoglio è un rivoluzionario che vede una Chiesa moderna e una modernità rivoluzionaria sia per la Chiesa sia per la società e per la costruzione di un dio unico che non è soltanto quello descritto e venerato dai cattolici e anche dai protestanti ma è unico al mondo anche se suscita nei popoli delle specifiche religioni (...) è una novità assolutamente unica di questo Pontefice che sta lavorando per una Chiesa che compie due funzioni contemporanee: costruire una Chiesa moderna dalla quale derivi una società al tempo stesso moderna e religiosa» (Scalfari 2020).

Il Pontefice pare essere riuscito, come capita ai grandi leader carismatici, a conquistarsi un'autorevole spazio di autonomia che propone, ai cattolici ma non solo, un percorso per attraversare la storia ed un orizzonte verso cui muoversi nella costruzione del mondo che verrà una volta superato il dramma collettivo che si sta vivendo ${ }^{13}$. Un Papa che, quando la Cei «esige» la riapertura dei luoghi di culto ${ }^{14}$, non ha problemi ad intervenire immediatamente, richiamando alla prudenza ed allobbedienza dei provvedimenti ed evitando il rischio di una contrapposizione frontale fra i vescovi italiani e le direttive statuali di avvio della seconda fase di governo della pandemia. ${ }^{15}$ Pochi giorni dopo, il 7 maggio, il

\footnotetext{
${ }^{12}$ È il richiamo alle situazioni di indigenza, alla condizione dei detenuti, alla richiesta di tregua nei conflitti armati. ${ }^{13}$ Sulla figura di Francesco si vedano i contributi raccolti in Garelli e Pace, 2016.

${ }^{14}$ La nota della Cei a seguito del Dpcm del 26 aprile che tracciava le linee di passaggio alla fase 2 di parziale allentamento dei vicoli è particolarmente dura: «Alla Presidenza del Consiglio e al Comitato tecnico-scientifico si richiama il dovere di distinguere tra la loro responsabilità - dare indicazioni precise di carattere sanitario - e quella della Chiesa, chiamata a organizzare la vita della comunità cristiana, nel rispetto delle misure disposte, ma nella pienezza della propria autonomia. I Vescovi italiani non possono accettare di vedere compromesso l'esercizio della libertà di culto. Dovrebbe essere chiaro a tutti che l'impegno al servizio verso i poveri, così significativo in questa emergenza, nasce da una fede che deve potersi nutrire alle sue sorgenti, in particolare la vita sacramentale». (Conferenza Episcopale Italiana, 2020b)

15 «In questo tempo nel quale si comincia ad avere disposizioni per uscire dalla quarantena, preghiamo il Signore perché dia al suo popolo, a tutti noi, la grazia della prudenza e della obbedienza alle disposizioni, perché la pandemia non torni». (Francesco, 2020b)
} 
Presidente della Cei, il Presidente del Consiglio e il Ministro dell'Interno sottoscrivono un protocollo d'intesa che consente, a partire dal 18 maggio, la graduale ripresa delle celebrazioni liturgiche con il popolo (Muolo, 2020).

\section{Considerazioni conclusive}

La storia si è interrotta. La cesura introdotta dalla pandemia segnerà la memoria collettiva e richiederà, dopo il periodo di convalescenza, la rimessa in discussione del vocabolario e della grammatica del linguaggio delle relazioni interpersonali. Ad evento in corso è difficile prevedere quanto e come i sistemi di valori e i sistemi di interessi si rimoduleranno nella costruzione di un nuovo ordine mondiale. A fine Ottocento la dottrina sociale della Chiesa nasceva come risposta alle Rerum novarum, alle cose nuove di un mondo in tumultuoso cambiamento, oggi la stessa sollecitudine imporrà una strategia di adattamento dell'istituzione alle Rerum novarum che struttureranno la convivenza sociale post frattura.

In Italia l'esplosione della pandemia ha accentuato i punti di forza e quelli di debolezza della presenza della Chiesa cattolica. In un primo momento, accentuando gli elementi di irrilevanza del «peso politico» della funzione sociale svolta dalla religione, il mondo cattolico è apparso afono e subalterno a decisori e decisioni che hanno considerato le attività ordinarie della Chiesa al pari di quelle di tanti altri ambienti sociali entro cui si sperimentano momenti di socialità condivisa. I comunicati della Cei di immediato e pedissequo accoglimento dei $\mathrm{Dpcm}$ sono apparsi a tanti come un segnale di forte riduzione dei livelli di autonomia nelle scelte di una libera Chiesa che vive dentro un libero Stato. Segnali della secolarizzazione (servizi religiosi non privilegiabili rispetto ad altri servizi), segnali di una sfiducia nella capacità di autoregolamentazione delle attività religiose, segnali di sospensione di pratiche che accentuano e legittimano le già diffuse tendenze di individualismo religioso e di credenze in cui il percorso di salvezza self service può fare a meno della frequentazione di ambienti e risorse sacramentali di cui la Chiesa dispone. La Chiesa che uscirà dopo la pandemia dovrà adattarsi a un mondo nuovo e presumibilmente disorientato. La Chiesa cattolica nella sua millenaria storia è sempre stata capace di innovarsi. A volte lo ha fatto grazie alla proposizione di spinte che venivano dal basso, con i nuovi movimenti ecclesiali. Questa pare essere la volta in cui, se ci sarà, la spinta proverrà dall'alto della sua gerarchia. In casi come questo il successo del processo di innovazione dovrà vincere le resistenze che saranno poste non tanto dall'ambiente sociale nel suo complesso, ma dall'interno della stessa Chiesa. Papa Francesco con la sua Chiesa «in uscita» chiede l'apertura del recinto e sollecita i cattolici a «stare» nella realtà, anche quella più scomoda, con l'attesa che l'incontro con la realtà renda l'esperienza di fede capace di contaminarsi col concreto vissuto dei singoli e dei gruppi sociali. In questo percorso, traspare una sostanziale continuità fra Benedetto XVI e Francesco: «Il primo ha posto con grande lucidità la questione europeo-cristiano-occidentale: il restringimento della ragione che pone fine a unalleanza millenaria. Il pontefice argentino, invece, insiste nel far presente a noi europei che non si riallarga la ragione attraverso la ragione. Ma solo tornando a sporcarsi le mani con l'uomo in carne e ossa, con la nuda vita là dove pulsa. Facendosi interrogare e rimettere in movimento da tutto ciò che il modello che abbiamo costruito (fondato appunto sull'astrazione) lascia fuori. Questo vuol dire, per la Chiesa, accettare di 
farsi scomodare. Uscire dalle cattedrali, dalle sagrestie, dai libri, dai convegni, dalle proprie case, dalle istituzioni per andare e toccare, con tenerezza, quella realtà dalla quale le forme sociali, per il modo in cui si sono organizzate, ci tengono lontani» (Giaccardi, Magatti, 2019, p. 134-135). Papa Francesco sembra dare per acquisita e consolidata la dottrina e, piuttosto che disegnare confini, o affidarsi, a inconciliabili dispute teologiche, pare volere tornare alle radici del fatto cristiano proponendolo come "primo annuncio" a un contesto in cui l'annuncio è ignoto o talmente incrostato da renderne ormai invisibile l'essenza. È l'incontro con la concretezza della realtà che può rendere capace, l'ormai "piccolo resto di Israele», di dare una ragionevole e plausibile giustificazione del proprio credere. Il «noi» cui guarda Papa Bergoglio è necessariamente plurale: è il complicato compito di sostenere la nascita di carismi che vivano un nuovo tipo di ortodossia, non più sostenuta dalla forza sociale della tradizione e da un rigido apparato di controllo di confini e metodi (Pace, 2012). Intervistato nei giorni più drammatici della pandemia, Francesco esplicita un'immagine di Chiesa nuova che si sottrae al dibattito ideologico fra istituzione e carisma: «Meno aggrappata alle istituzioni? Direi piuttosto agli schemi. Infatti la Chiesa è istituzione. Esiste la tentazione di sognare una Chiesa de-istituzionalizzata, per esempio una Chiesa gnostica, senza istituzioni, o soggetta a istituzioni fisse, per proteggersi, ed è una Chiesa pelagiana. A rendere la Chiesa istituzione è lo Spirito Santo. Che non è gnostico né pelagiano. È lui a istituzionalizzare la Chiesa. È una dinamica alternativa e complementare, perché lo Spirito Santo provoca disordine con i carismi, ma in quel disordine crea armonia. Chiesa libera non vuol dire una Chiesa anarchica, perché la libertà è dono di Dio. Chiesa istituzionalizzata vuol dire Chiesa istituzionalizzata dallo Spirito Santo. Una tensione tra disordine e armonia: è questa la Chiesa che deve uscire dalla crisi. Dobbiamo imparare a vivere in una Chiesa in tensione tra il disordine e l'armonia provocati dallo Spirito Santo. Se mi chiede un libro di teologia che possa aiutarla a comprenderlo, sono gli Atti degli apostoli. Ci troverà il modo in cui lo Spirito Santo de-istituzionalizza quello che non serve più e istituzionalizza il futuro della Chiesa. Questa è la Chiesa che deve uscire dalla crisi» (Ivereigh, 2020). ${ }^{16} \mathrm{E}$ un passaggio di fase che tenta di armonizzare la velocità del mutamento sociale alla plausibilità della proposta di fede cattolica. La sfida è di rovesciare la constatazione che le ragionevolezze della modernità avanzata hanno staccato in plausibilità quelle offerte della religione di chiesa. La pandemia ha cambiato il corso della storia e richiederà un generalizzato processo di risocializzazione. Un nuovo post in questi tempi tumultuosi, dopo la società post-industriale, post-moderna, post-materialista, post-comunista e post-verità. Un processo in cui la domanda di senso avrà bisogno di incontrare risposte adeguate a fornire orientamenti fra legature ed opzioni (Dahrendorf, 1994) per attraversare i tempi del post epidemia.

\section{Bibliografia}

Abbruzzese, S. (2010). Un moderno desiderio di Dio. Ragioni del credere in Italia. Soveria Mannelli: Rubbettino.

Agesci, (2020). Comunicazione a tutti i livelli associativi. In www.agesci.it, 10 marzo. Berger, P. (1984). La sacra volta. Milano: Sugarco.

\footnotetext{
${ }^{16}$ Sul tema della relazione fra carisma ed istituzione si veda Ratzinger, 1999.
} 
Berger, P. (1987). L'imperativo eretico. Leumann(To): Elle Di Ci.

Berger, P. (2017). I molti altari della modernità. Le religioni al tempo del pluralismo. Bologna: Emi.

Berzano, L. (2014). Quarta secolarizzazione. Milano-Udine: Mimesis.

Berzano, L. (2020. Il religioso smaterializzato. Hermeneutica. Annuario di filosofia e teologia.

Bova, V. (2014). Cattolicesimi d'Italia. Un'identità religiosa. Roma: Carocci.

Carron, J. (2020). Lettera alla fraternità di Comunione e liberazione. In: it.clonline.org, 12 marzo.

Casanova, J. (2000). Oltre la secolarizzazione. Le religioni alla riconquista della sfera pubblica. Bologna: il Mulino.

Conferenza Episcopale Italiana (2020). Decreto "Coronavirus". La posizione della CEI, in www.ceinews.it, 8 marzo.

Conferenza Episcopale Italiana (2020a). Una chiesa di terra e di cielo, in www.chiesacattolica.it.

Conferenza Episcopale Italiana (2020b). Dpcm, la posizione della Cei, in chiesacattolica.it, 26 aprile.

Conte, G. (2020). Conte: grazie alla Chiesa. Giorni cruciali, in Avvenire 11 aprile.

Dahrendorf, R.(1994). La libertà che cambia. Roma-Bari: Laterza.

Diamanti, I. (2020). Se il virus ridisegna i nostri confini. In: Repubblica 30 marzo.

Direzione nazionale Acli (2020). Vicini a distanza. La Direzione Nazionale Acli sullemergenza coronavirus, in www.acli.it, 11 marzo.

Durkheim, È. (1973). Le forme elementari della vita religiosa. Roma: Newton Compton.

Francesco (2015). Lettera enciclica Laudato si. In: www.vatican.va.

Francesco (2020). Omelia, Sagrato della Basilica di San Pietro. In: www.vatican.va, 27 marzo.

Francesco (2020a). Omelia, Cappella Santa Marta. In: www.vatican.va, 29 marzo.

Francesco (2020b). Omelia, messa Santa Marta. In: www.vaticannews.va, 28 aprile.

Franco, M. (2020). Il Papa, il Coronavirus e il dietrofront sulle chiese chiuse. I vescovi italiani e quei malumori captati da Francesco. In: Corriere della sera, 15 marzo.

Garelli, F. (2020). Gente di poca fede. Il sentimento religioso nell'Italia incerta di Dio. Bologna: il Mulino.

Garelli, F. (2020a). Funzioni religiose sospese il 68\% degli italiani d'accordo. In: Il Messaggero 30 marzo.

Garelli, F. e Pace, E. (2016). Sfide per il nuovo papato. Studi sociologici. Numero monografico di Rassegna italiana di sociologia, 4.

Giaccardi, C. e Magatti, M. (2019). La scommessa cattolica. Bologna: il Mulino.

Giovanni Paolo II (1987). Lettera enciclica Sollicitudo rei socialis. In: www.vatican.va.

Gnagni, F. (2020). L'Europa ritrovi lo spirito cristiano. Parla Salvatore Martinez (RNS). In: Formiche.net, 13 aprile.

Hervieu-Léger, D. (2003). Il pellegrino e il convertito. La religione in movimento. Bolgna: il Mulino.

Ivereigh, A. (2020). Intervista a papa Francesco. Il Papa: cosi sto vivendo l'emergenza pandemia. In: www.vaticannews.va 8 aprile.

Le Bon, A. (2014). Psicologia delle folle. Massa (Ms): Ed. Clandestine. 
Movimento dei focolari (2020). Emergenza coronavirus. In: www.focolare.org/ news/2020/02/22/

Muolo, M. (2016). Papa Francesco e l'enciclica dei gesti. In: www.avvenire.it 15 giugno.

Muolo, M. (2020). Firmato il protocollo: messe aperte ai fedeli dal 18 maggio. Ecco come saranno. In: Avvenire.it, 7.05.2020.

Ognibene, F. (2020). Coronavirus. Associazioni e parlamentari cattolici: noi col Papa, vicini ai vescovi. In: Avvenire 16 marzo.

Pace, E. (2012). Il carisma, la fede, la chiesa. Roma: Carocci.

Paolo VI (1967). Lettera enciclica Populorum progressio. In: www.vatican.va.

Penitenzieria Apostolica (2020). Decreto della Penitenzieria Apostolica circa la concessione di speciali Indulgenze ai fedeli nell'attuale situazione di pandemia. In: press.vatican.va 20 marzo.

Politi, M. (2020). Coronavirus, svanisce la religione: al suo posto regna la scienza. Persino il Papa se nè accorto. In: Il fatto Quotidiano 26 marzo.

Pontificio Consiglio della Giustizia e della Pace (2004). Compendio della dottrina sociale della Chiesa. Città del Vaticano: Libreria editrice vaticana.

Presidenza e Consiglio nazionale Ac (2020). Responsabilità, vicinanza, preghiera e gratitudine. In: www.azionecattolica.it, 16 marzo.

Ratzinger, J. (1999). Relazione introduttiva in Pontificium consilium pro laicis, I movimenti nella Chiesa. Atti del congresso mondiale dei movimenti ecclesiali, Roma, 27-29 maggio 1998, Città del Vaticano.

Rémond, R. (2003). La secolarizzazione. Religione e società nell'Europa contemporanea. Bari-Roma: Laterza.

Riccardi, A. (2020). Se per battere la paura del contagio si mettono in ginocchio le nostre chiese. La Stampa, 29 febbraio.

Roy, O. (2019). L'Europa è ancora cristiana? Milano: Feltrinelli.

Scalfari, E. (2020), La parola del Papa e la proposta di Draghi, in Repubblica 28 marzo.

Taylor, C. (2009). L'età secolare. Milano: Feltrinelli.

Tanzella-Nitti, G. (2020). Scienza e fede al tempo del Coronavirus, in Disf.org 21 marzo.

Ventura, R. A. (2020). Coronavirus, l'allarme di Ilaria Capua: in Lombardia sta succedendo qualcosa che non si spiega. In: www.fanpage.it, 20 marzo.

Weber, M. (1999). Economia e società, vol. II. Torino: Edizioni di Comunità. 